\title{
Is Bovine Heparin, an Old Fellow, a Safe Anticoagulation Approach during Extracorporeal Circulation Inside the Cardiac Operating Room?
}

Ivis Levy Fernandes Martins ${ }^{\circledR}$ and Christianne Bretas Vieira Scaramello

Universidade Federal Fluminense, Niterói, $R J$ - Brazil

Antithrombotic agents include anticoagulants, antiplatelet and fibrinolytic drugs. They are prescribed in several situations related to hemostasis disturbances that favor thrombi formation. Since bleeding is a common adverse effect of these drugs, a cost-benefit analysis of antithrombotic therapy prior to surgery should be performed. ${ }^{1}$ In order to consider the preoperative therapy for patients undergoing cardiac surgery, the risk of thrombosis must outweigh the risk of bleeding. Nonetheless, the literature has reported the use of anticoagulant therapy with unfractionated heparin (UFH) during extracorporeal circulation (ECC) inside and outside the cardiac operating room. ${ }^{1-4}$

The development of the heart-lung machine made ECC possible. Therefore, heart surgery became a routine practice. The literature describes that complications related to ECC have diminished due to modern monitoring, highly trained staff, special cannulation methods, as well as the latest advances in techniques. ${ }^{2}$ However, some authors suggest that guidelines based on more researches would improve the safety of this procedure. ${ }^{3}$ During cardiac surgery, ECC preserves the functional characteristics of the heart, provides a clean surgical environment and offers safety to the team. ${ }^{4}$

Heparin is a sulfated polysaccharide. It was the first anticoagulant drug approved and is one of the oldest in clinical use, being the second most widely used pharmaceutical drug by mass. Glycosaminoglycan heparin is a natural compound processed to pharmaceutical grade heparin, which can undergo controlled depolymerization to produce

\section{Keywords}

Heparin; Blood Coagulation; Extracorporeal Circulation; Cardiac Surgical Prcedures; Patient Safety. low-molecular-weight heparins (LMWHs), whose molecular weights are approximately one-third that of the parent heparin. UFH binds with high affinity to antithrombin, increasing its ability to inhibit both factor Xa and thrombin in the coagulation cascade, whilst LMWHs primarily inhibit factor $\mathrm{Xa}$, and are used therapeutically because of their improved pharmacokinetics and reduced side effects over UFH. ${ }^{5,6}$

UFH has also been manufactured from different mammalian sources, such as porcine (pig), bovine (cow) and ovine (sheep) tissues. Nowadays, there are some concerns about a shortage of porcine heparin. Thus, regulatory agencies are currently considering the introduction of bovine UFH for parenteral indications, and ovine heparin is being developed in non-US markets. ${ }^{6}$ For this reason, recent studies have discussed the use of bovine UFH in cardiac surgery. ${ }^{7,8}$

The paper published by Torres et al., ${ }^{7}$ in the International Journal of Cardiovascular Sciences, reports the safety of bovine UFH evaluated during cardiac pump surgery. The study included a retrospective and descriptive analysis. The authors analyzed the medical records of all patients undergoing cardiac surgery with ECC using bovine UFH (supplied by Eurofarma Laboratories S.A.), between October 2008 and November 2012, and porcine UFH (supplied by Cristália and Blausiegel Laboratories), between June 2013 and December 2014, at the Heart Surgery Institute of Hospital Bom Jesus in Ponta Grossa (Paraná, Brazil). Patients were excluded from the study if they had received UFH, LMWH, oral anticoagulants and/or fibrinolytics before the procedure. They were also excluded in case of absence of preoperative platelet count and/or cardiac surgery performed in the presence of severe sepsis, with high risk of widespread intravascular coagulation. Pre- and postoperative

Mailing Address: Christianne Bretas Vieira Scaramello

Rua Prof. Hernani Mello, 101. Postal Code: 24210-130, Niterói, RJ - Brazil.

E-mail: chrisbretas@gmail.com

DOI: https://doi.org/10.36660/ijcs.20200077 
data were also collected. The study outcomes were: bleeding, thrombocytopenia, postoperative vasoplegia, activated clotting time shorter than 400 seconds and coagulation phenomena, such as blood clotting with bovine UFH (data on porcine UFH were also collected only as exploratory means).

Torres et al., ${ }^{7}$ evaluated 204 medical records of eligible patients who used bovine UFH. Out of these patients, $66.18 \%$ presented thrombocytopenia, whereas $1.04 \%$ presented bleeding of more than $2000 \mathrm{~mL}$, within the first 24 hours of the postoperative period. Only one patient presented clots in the surgical field. Median activated clotting time increased five-fold after the first dose of heparin and, after protamine, it returned to similar baseline values. Based on these findings, the authors have concluded that bovine UFH does not present unusual adverse effects and can be considered safe for on-pump cardiac surgery.

Gomes et al., ${ }^{8}$ have also published a paper on a preliminary study evaluating the safety and important

\section{References}

1. Kreuziger LB, Karkouti K, Tweddel J, Massicotte MP. Antithrombotic therapy management of adult and pediatric cardiac surgery patients. J Thromb Haemost. 2018;16(11):2133-46

2. Feindt P, Benk C, Boeken U, Bauer A, Mehlhorn U, Gehron I, et al. Use of extracorporeal circulation (ECC) outside the cardiac operating room: indications, requirements and recommendations for routine practice. Thorac Cardiovasc Surg. 2011;59(2):66-8.

3. Lukaszewski M, Kosiorowska K, Kościelska-Kasprzak K, Jakubaszko J, Bielicki G, Jasiński M. The use of modern monitoring techniques and methodologies in conducting extracorporeal circulation: a place for Quantum Heart Lung Machine. Kardiol Pol. 2019;77(6):642-4.

4. Torrati FG, Dantas RAS. Extracorporeal circulation and complications during the immediate postoperative period for cardiac surgery. Acta Paul Enferm. 2012; 25(3):340-5. clinical aspects of bovine UFH use in heart surgery and compared it to porcine heparin, both obtained from intestinal mucosa. Data were extracted from medical records as well. The main finding of this work was in accordance to Torres et $\mathrm{al}^{7}$, as similar data were obtained from bovine and porcine UFH treated patients. They have evaluated bleeding volume, modulation of clotting, presence of clot in the surgical field during ECC, activated partial thromboplastin time and specific anti-Xa anticoagulant activity. The authors concluded that bovine UFH may be the solution for the tainted heparin supply.

Although the paper presented by Torres et al., ${ }^{7}$ has limitations consistent with retrospective studies, it is an important contribution to the field. The use of bovine $\mathrm{UFH}$ is on the rise due to contamination of porcine UFH with oversulfated chondroitin sulfate, which has caused serious adverse effects. Therefore, studies that corroborate the safety of bovine UFH are welcome.

5. Devlin A, Mycroft-West C, Procter P, Cooper L, Guimond S, Lima M, et al. Tools for the quality control of pharmaceutical heparin. Medicina. 2019; 55(10):1-19.

6. Kouta A, Jeske W, Hoppensteadt D, Iqbal O, Yao Y, Fareed J. Comparative pharmacological profiles of various bovine, ovine, and porcine heparins. Clin Appl Thromb Haemost. 2019;25:1-9.

7. Torres FAL, Torres ACB, Ribeiro A, Maia CO, Almeida FP, Roceto J, et al. A Retrospective Study on Unfractionated Bovine Heparin Safety in On-Pump Cardiac Surgery. Int J Cardiovasc Sci. 2020; 33(3):235-242.

8. Gomes WJ, Leal JC, Braile DM, Guimarães JA, Lopes RD, Lima MA, et al. A Brazilian perspective for the use of bovine heparin in open heart surgery. Int J Cardiol. 2016;15(223):611-2. 